\title{
Serum Uric Acid, Hemoglobin A1c, Glucose Metabolism Abnormalities and Early Stage Atherosclerosis in Chinese Adults-The Cardiometabolic Risk in Chinese (CRC) Study
}

\author{
Qinqin Qiu ${ }^{1-3}$ and Jun Liang ${ }^{1-3^{*}}$ \\ ${ }^{1}$ Department of Endocrinology, Xuzhou Central Hospital, Xuzhou Clinical School of Xuzhou Medical College, Affiliated Hospital of Southeast University, Xuzhou, Jiangsu, \\ China
}

${ }^{2}$ Xuzhou Institute of Medical Sciences, Xuzhou, Jiangsu, China

${ }^{3}$ Xuzhou Institute of Diabetes, Xuzhou, Jiangsu, China

*Corresponding author: Dr. Jun Liang, Department of Endocrinology, Xuzhou Central Hospital, 199\# South Jiefang Road, Xuzhou, Jiangsu, China, 221009, Fax: +86-83840486; Tel: +86-18952171209; E-mail: mwlj521@163.com

Received date: December 22, 2015; Accepted date: April 17, 2016; Published date: April 27, 2016

Copyright: $\odot 2016$ Qiu Q, et al. This is an open-access article distributed under the terms of the Creative Commons Attribution License, which permits unrestricted use, distribution and reproduction in any medium, provided the original author and source are credited.

\section{Letter to Editor}

We are grateful to your keen interest in the study "Adiponectin Genotype, Blood Pressures, and Arterial Stiffness: The Cardiometabolic Risk in Chinese (CRC) Study" published recently in Journal of Clinical Hypertension.

Atherosclerotic cardiovascular disease has been one of the leading causes of death in modern society. Pulse wave velocity (PWV) is a gold standard for assessing arterial stiffness and widely used indicator for early atherosclerosis [1,2]. PWVs may reflect the atherosclerotic alterations at central (cf-PWV) or peripheral arteries (cd-PWV and crPWV). Several previous studies have shown that hyperuricemia and long-term glycemic exposure were associated with cardiovascular disease [3-5] and the adverse effects of serum uric acid (SUA) may occur at early stage of atherosclerosis [6-8]. The effects of various glucose exposures are not perfectly concordant and may be independent. However, the available data are very limited on the relationship between SUA, hemoglobin Alc, glucose metabolism abnormalities and pulse wave velocity, an index of early arterial stiffness.

In the Cardiometabolic Risk in Chinese (CRC) Study, we performed a community-based health examination survey for subjects (29-79 y) who were randomly selected from residents living in the urban area of Xuzhou, China, in 2009. All subjects were successfully measured for PWVs, blood pressure, body mass index (BMI), heart rate (HR), SUA and other metabolic markers. PWV was based on the distance/time ratio (meters/second), was calculated as the path length divided by the transit time and expressed as $\mathrm{m} / \mathrm{s}$. In a cross-sectional study, it was found that SUA was independently associated with brachial ankle PWV (baPWV) in She Ethnic Minority Group of Fujian province in China [9]. However, baPWV is an index of both central and peripheral arterial stiffness index [10]. It is not clear whether SUA levels specifically affect central or peripheral arterial stiffness. In our study of a large sample of Chinese adults, we found that SUA levels were significantly related to central arterial stiffness independent of conventional risk factors, such as sex, BMI, lipids, glucose metabolism [11]. Whereas SUA levels were not associated with peripheral arterial stiffness, measured by cr-PWV and cd-PWV. Intriguingly, the association between SUA and cf-PWV was stronger among adults with higher HR ( $\geq 65 \mathrm{bpm})$. Several cohort studies have found that a synergistic role of high baseline HR and changes in HR during the follow-up period in accelerating increases of PWV [12-14]. The results of our study were in accordance with those of several investigations that have shown increased HR at rest was a significant risk factor for CVD and a marker of new onset of atherosclerosis [15]. Nevertheless, a recent study in Germany found that decreased heart rate variability correlates to increased cardiovascular risk [16]. In addition, we found significant interaction between hypertension status and SUA level in relation to cf-PWV. In a previous prospective study, it was found that that inflammatory and adiponectin-mediated proatherogenic activation are interrelated, which interact leading to a significant increase of arterial stiffness in essential hypertensive patients [17]. However, the mechanisms underlying such an additive effect remain not clear. High SUA levels might play a more important role in arterial stiffness before the development of hypertension and the adverse effects of SUA on central arterial stiffness appeared more evident in people with normal blood pressure than those with hypertension. This finding might have important clinical implications for prevention and intervention of cardiovascular risk at early stage. Also, age might modify the deleterious effects of SUA. Our results suggest that older individuals may be more sensitive to the detrimental effects of high SUA on cf-PWV. However, there was no significant interactions of SUA with sex and BMI in relation to cf-PWV.

Some recent studies found that abnormal glucose metabolism was related to increased risk of cardiovascular diseases [18]. Also HbAlc was associated with arterial stiffness measured by cf-PWV in patients with type 2 diabetes [19-21]. But the studies examining the relation between HbAlc and arterial stiffness in the general population were sparse and little was known about the relative influence of $\mathrm{HbAlc}$ and fasting and post-challenge glucose on cardiovascular risk. Few studies have examined whether glucose metabolism abnormalities affect early atherosclerosis in Chinese.

In CRC study, a linear regression model was used to evaluate associations between glucose exposures ( $\mathrm{HbAlc}$, fasting glucose, and $2 \mathrm{~h}$ OGTT) and cf-PWV, adjusting for covariates. Our results indicated that HbAlc was related to cf-PWV, independent of other conventional cardiovascular risk factors and showed additive effects with fasting glucose or $2 \mathrm{~h}$ OGTT on cf-PWV [22]. Yuqian Bao et al. found that $\mathrm{HbAlc}$ reflect the risk of subclinical atherosclerosis in middle-aged and elderly Chinese populations with impaired glucose regulation [23]. Individuals of both high HbAlc and IFG or IGT had significantly higher levels of cf-PWV compared with those who only had high HbAlc. The associations were stronger in subjects who were older $(\geq$ $60 \mathrm{y})$ and had higher blood pressure $(\geq 120 / 80 \mathrm{mmHg})$ than those who were younger and had lower blood pressure. Our findings of the 
additive effects of different measures of glucose exposures highlight the importance to measure $\mathrm{HbAlc}$ in addition to fasting glucose and $2 \mathrm{~h}$ OGTT in characterizing individuals at high risk of cardiovascular disease. In addition, cf-PWV was associated with the risk of IFG, independent of other metabolic risk factors, and the association was modified by age and neck circumference levels. The association between cf-PWV and IFG was stronger in participants who were younger $(\mathrm{p}<0.01)$, which was not the same with previous research. The association was significant in the group with a high level of neck circumference levels. These findings suggested that the changes of atherosclerosis might have started since early prediabetes stage.

About the association of early atherosclerosis with different glucose metabolism status, we had done some other studies. Alterations in vascular structure and function, including increased wall thickness, as indexed by intima-media thickness (IMT) and decreased arterial wall elasticity, are increasingly recognized as significant independent predictors of adverse cardiovascular outcomes [24-26]. All subjects were divided into five groups: normal glucose tolerance group (NGT), impaired fasting glucose group (IFG), impaired glucose tolerance group (IGT), IFG + IGT group and newly diagnosed type 2 diabetes mellitus group (PDM). General clinical data of subjects in each group were collected, and biochemical indicators were measured. We found that IMT values increased significantly in IFG, IGT, IFG + IGT and PDM group as compared with NGT group $(\mathrm{P}<0.01)$. With the worsening of abnormal glucose metabolism, from IFG, IGT to IFG + IGT, the IMT values increased gradually, but there was no difference among the three groups. According to multivariate stepwise regression analysis, age, fasting plasma glucose, fasting insulin and insulin resistance index were independent risk factors of IMT. We concluded that the IMT increased significantly in IFG, IGT, IFG + IGT and PDM group, which showed that the change of atheroselerosis was associated with early prediabetes stage.

The sample size of the present study was large, which ensured sufficient power to detect the moderate effects of SUA, HbAlc, glucose metabolism abnormalities on arterial stiffness and interactions with other cardiometabolic risk factors. However, several limitations of this study warrant consideration. Due to the cross-sectional nature of this study, making causal interpretations of associations between SUA, HbAlc, glucose metabolism abnormalities and risk of cardiovascular disease difficult, we cannot determine the temporality of the observed associations. In our study samples, we did not collection information of dietary intake and the lifestyle information is not incomplete. Therefore, it is still possible the residual confounding of these uncollected variables might influence the results. The inflammatory markers and cytokines were also not included. In addition, the study was performed in a Chinese population. Further studies in other populations of different ethnicities are warranted to verify our findings. In the future, It is important to prevent and intervene cardiovascular risk factors (eg. SUA, glucose, blood pressure, HR) at early stage of atherosclerosis.

\section{References}

1. Mitchell GF, Hwang SJ, Vasan RS, Larson MG, Pencina MJ, et al. (2010) Arterial stiffness and cardiovascular events: the Framingham Heart Study. Circulation 121: 505-511.

2. Reising A, Sambale S, Donnerstag F, Schmidt JJ, Hafer C, et al. (2013) Effect of isovolemic, isothermic hemodialysis on cerebral perfusion and vascular stiffness using contrast computed tomography and pulse wave velocity. PLoS One 8: e56396.
3. Tsioufis C, Kyvelou S, Dimitriadis K, Syrscloudis D, Sideris S, et al. (2011) The diverse associations of uric acid with low-grade inflammation, adiponectin and arterial stiffness in never-treated Hypertensives. J Hum Hypertens 25: 554-559.

4. Liu WC, Hung CC, Chen SC, Yeh SM, Lin MY, et al. (2012) Association of hyperuricemia with renal outcomes, cardiovascular disease, and mortality. Clin J Am Soc Nephrol 7: 541-548.

5. Teng F, Zhu R, Zou C, Xue Y, Yang M, et al. (2011) Interaction between serum uric acid and triglycerides in relation to blood pressure. J Hum Hypertens 25: 686-691.

6. Qin L, Yang Z, Bin L, Wen J, Zi Ye, et al. (2011) Serum uric acid level and its association with metabolic syndrome and carotid atherosclerosis in patients with type 2 diabetes. Cardiovascular Diabetology 10: 72.

7. Rodrigues TC, Maahs DM, Johnson RJ, Jalal DL, Kinney GL, et al. (2010) Serum Uric Acid Predicts Progression of Subclinical Coronary Atherosclerosis in Individuals Without Renal Disease. Diabetes Care 33: 2471-2473.

8. Hashemi M, Yavari M, Amiri N, Taheri H, Shaigannia I, et al. (2007) Uric acid: a risk factor for coronary atherosclerosis? Cardiovasc J South Afr 18: 16-19.

9. Lin Y, Lai X, Chen G, Xu Y, Huang B, et al. (2012) Association among serum uric acid, cardiovascular risk, and arterial stiffness: a crosssectional study in She ethnic minority group of Fujian Province in China. J Endocrinol Invest 35: 290-297.

10. Lee JY, Ryu S, Lee SH, Kim BJ, Kim BS, et al. (2015) Association between brachial-ankle pulse wave velocity and progression of coronary artery calcium: a prospective cohort study. Cardiovasc Diabetol 14: 147.

11. Liang J, Li Y, Zhou N, Teng F, Zhao J, et al. (2012) Synergistic effects of serum uric acid and cardiometabolic risk factors on early stage atherosclerosis: the cardiometabolic risk in Chinese study. PLoS One 7: e51101.

12. Tomiyama $\mathrm{TH}$, Hashimoto $\mathrm{H}$, Tanaka $\mathrm{H}$, Matsumoto $\mathrm{C}$, Odaira $\mathrm{M}$, et al. (2010) Brachial ankle PWV Collaboration Group. Synergistic relationship between changes in the pulse wave velocity and changes in the heart rate in middle-aged Japanese adults: a prospective study. J Hypertens 28: 687-694.

13. Wang L, Zhang N, Sun BG, Wang Z, Cao ZY (2016) Elevated Resting Heart Rate Is Associated with Carotid Atherosclerosis in Middle-Aged and Elderly Chinese Population. J Stroke Cerebrovasc Dis 25: 34-40.

14. Simula S, Vanninen E, Lehto S, Hedman A, Pajunen P, et al. (2014) Heart rate variability associates with asymptomatic coronary atherosclerosis. Clin Auton Res 24: 31-37.

15. Fox K, Borer JS, Camm AJ, Danchin N, Ferrari R, et al. (2007) Resting heart rate in cardiovascular disease. J Am Coll Cardiol 50: 823-830.

16. Schuster AK, Fischer JE, Thayer JF, Mauss D, Jarczok MN (2016) Decreased heart rate variability correlates to increased cardiovascular risk. Int J Cardiol 203: 728-730.

17. Tsioufis C, Dimitriadis K, Selima M, Thomopoulos C, Mihas C, et al. (2007) Low-grade inflammation and hypoadiponectinaemia have an additive detrimental effect on aortic stiffness in essential hypertensive patients. Eur Heart J 28: 1162-1169.

18. Rasmussen-Torvik LJ, Li M, Kao WH, Couper D, Boerwinkle E, et al. (2011) Association of a fasting glucose genetic risk score with subclinical atherosclerosis: The Atherosclerosis Risk in Communities (ARIC) study. Diabetes 60: 331-335.

19. Chen Y, Huang Y, Li X, Xu M, Bi Y, et al. (2009) Association of arterial stiffness with $\mathrm{HbAlc}$ in 1,000 type 2 diabetic patients with or without hypertension. Endocrine 36: 262-267.

20. Zieman SJ, Kamineni A, Ix JH, Barzilay J, Djoussé L, et al. (2012) Hemoglobin Alc and arterial and ventricular stiffness in older adults. PLoS One 7: e47941.

21. Bower JK, Appel LJ, Matsushita K, Young JH, Alonso A, et al. (2012) Glycated hemoglobin and risk of hypertension in the atherosclerosis risk in communities study. Diabetes Care 35: 1031-1037. 
Citation: Qiu Q, Liang J (2016) Serum Uric Acid, Hemoglobin A1c, Glucose Metabolism Abnormalities and Early Stage Atherosclerosis in Chinese Adults-The Cardiometabolic Risk in Chinese (CRC) Study. J Hypertens 5: 220. doi:10.4172/2167-1095.1000220

Page 3 of 3

22. Liang J, Zhou N, Teng F, Zou C, Xue Y, et al. (2012) Hemoglobin Alc levels and aortic arterial stiffness: the Cardiometabolic Risk in Chinese (CRC) study. PLoS One 7: e38485.

23. Ma X, Shen Y, Hu X, Hao Y, Luo Y, et al. (2015) Associations of glycated haemoglobin A1c and glycated albumin with subclinical atherosclerosis in middle-agedand elderly Chinese population with impaired glucose regulation. Clin Exp Pharmacol Physiol 42: 582-587.

24. Inaba Y, Chen JA, Bergmann SR (2011) Carotid intima-media thickness and cardiovascular events. N Engl J Med 365: 1640-1641.
25. Frimodt-Møller M, Kamper AL, Strandgaard S, Kreiner S, Nielsen AH (2012) Beneficial effects on arterial stiffness and pulse-wave reflection of combined enalapril and candesartan in chronic kidney disease--a randomized trial. PLoS One 7: e41757.

26. Niu L, Zhang Y, Qian M, Meng L, Xiao Y, et al. (2013) Impact of multiple cardiovascular risk factors on carotid intima-media thickness and elasticity. PLoS One 8: e67809. 